\title{
X-ray reflectivity study of temperature-dependent surface layering in liquid $\mathbf{H g}$
}

\author{
E. DiMasi* \\ Department of Physics, Brookhaven National Laboratory, Upton, New York 11973-5000 \\ H. Tostmann \\ Division of Engineering and Applied Sciences and Department of Physics, Harvard University, Cambridge, Massachusetts 02138 \\ B. M. Ocko \\ Department of Physics, Brookhaven National Laboratory, Upton, New York 11973-5000 \\ P. S. Pershan \\ Division of Engineering and Applied Sciences and Department of Physics, Harvard University, Cambridge, Massachusetts 02138 \\ M. Deutsch \\ Department of Physics, Bar-Ilan University, Ramat-Gan 52100, Israel
}

(Received 26 August 1998)

\begin{abstract}
We report x-ray reflectivity measurements of liquid mercury between $-36{ }^{\circ} \mathrm{C}$ and $+25{ }^{\circ} \mathrm{C}$. The surface structure can be described by a layered density profile convolved with a thermal roughness $\sigma_{T}$. The layering has a spacing of $2.72 \AA$ and an exponential decay length of $5.0 \AA$. Surprisingly, $\sigma_{T}$ is found to increase considerably faster with temperature than the $\sqrt{T}$ behavior predicted by capillary wave theory, in contrast with previous measurements on Ga and dielectric liquids. [S0163-1829(98)52144-3]
\end{abstract}

The effect of temperature on the surface structure of a liquid is quite different from that of a solid surface. In the liquid, thermal surface waves are excited at all wavelengths from the particle spacing to a long wavelength gravitational cutoff, and produce a surface roughness on the order of the particle spacing. For nonmetallic liquids, these capillary waves broaden the liquid-vapor interface, which is a monotonically decreasing density profile with a width of several A. ${ }^{1}$

Metallic liquids exhibit a more complex surface structure in which the atoms are stratified parallel to the liquid-vapor interface in layers that persist into the bulk for a few atomic diameters. This layering arises from the strongly densitydependent nonlocal interionic potential ${ }^{2}$ and is unique to metallic liquids. Nevertheless, the effect of temperature is still expected to be principally in the form of capillary waves, which roughen the surface-normal profile and diminish the layering amplitude. X-ray reflectivity measurements have confirmed the existence of surface layering in liquid $\mathrm{Hg}^{3}$ $\mathrm{Ga},{ }^{4} \mathrm{In},{ }^{5}$ and several alloys. ${ }^{6,7}$ Models based on capillary waves as the only mechanism for surface roughening were found to describe the temperature-dependent layering of liquid $\mathrm{Ga}^{8}$ as well as diffuse scattering measured from the liquid In surface. ${ }^{5}$

In previous comparisons of the $\mathrm{x}$-ray reflectivity of liquid $\mathrm{Hg}$ and $\mathrm{Ga},{ }^{9}$ two important differences were identified. Although reflectivities for both metals exhibit quasi-Bragg peaks indicative of surface layering, the $\mathrm{Hg}$ data have a minimum at low-momentum transfer $\left(q_{z} \approx 0.6 \AA^{-1}\right)$ not found in Ga (see Fig. 2 in Ref. 9). To describe this minimum, the model for the surface structure had to be more complex than that of Ga. One successful model incorporated a low density region persisting a few $\AA$ into the vapor side of the interface.
This feature was taken to be intrinsic to $\mathrm{Hg}$. In addition, the room-temperature layering peak appeared to be broader for $\mathrm{Hg}$ than for $\mathrm{Ga}$, leading to the conclusion that in $\mathrm{Hg}$, surface layering decayed over a much shorter length scale.

Subsequent measurements yielded variations in this low$q_{z}$ minimum, prompting us to address the possible effect of impurities at the surface. An important difference between experiments on $\mathrm{Hg}$ and $\mathrm{Ga}$ is that due to the low vapor pressure, Ga can be measured under ultra-high-vacuum (UHV) conditions and cleaned in situ by argon sputtering, which is not possible for $\mathrm{Hg}$. In this work, we compare $\mathrm{Hg}$ measured in a reducing $\mathrm{H}_{2}$ atmosphere ${ }^{10}$ to measurements taken in a UHV compatible chamber having a very low oxygen partial pressure.

The present temperature-dependent study has an important advantage over previous room-temperature measurements. Near the melting point, the layering peak has a large amplitude and is easily distinguished from the region of the low $-q_{z}$ minimum. This allows a robust determination of the layering decay length that is not affected by the details of the models in the near-surface region. This is not the case at room temperature, where the shallow peak and pronounced minimum yield more ambiguous fits. We conclude in the present work that $\mathrm{Ga}$ and $\mathrm{Hg}$ have comparable layering decay lengths. This underscores the value of temperaturedependent measurements for comparisons of liquid metals' surface structure.

$\mathrm{X}$-ray reflectivity measurements ${ }^{1}$ were carried out using the liquid surface spectrometer at beamline X22B at the National Synchrotron Light Source, at an X-ray wavelength of $1.24 \AA$ and resolution of $0.035 \AA^{-1} .11$ The background intensity, due mainly to scattering from the bulk liquid, was subtracted from the specular signal by rocking the detector out of the reflection plane. 
Liquid mercury was prepared in two different sample chambers. In the first, the $\mathrm{Hg}$ sample ${ }^{12}$ was contained in a glass reservoir with a Teflon stopcock and glass filling capillary, mounted on a high vacuum chamber comprised of a cylindrical thin-walled $\mathrm{Be}$ can mating to stainless steel flanges with viton o-ring seals. After several cycles of evacuating the chamber to $10^{-4}$ Torr and backfilling with 900 Torr of dry $\mathrm{H}_{2}$ gas, the liquid $\mathrm{Hg}$ was poured from the reservoir into a ceramic sample pan. Surfaces appeared clean to the eye and yielded reproducible reflectivity measurements for as long as 4 days. When the sample was deliberately exposed to air, the reflectivity exhibited deep oscillations characteristic of rapid oxide formation. ${ }^{13}$

A second $\mathrm{Hg}$ reservoir, capillary and valve made from stainless steel was mounted on top of a UHV chamber. Following a bakeout, the sample was poured, with the $\mathrm{Hg}$ vapor pressure of about $10^{-2}$ Torr then determining the total pressure in the chamber. Small patches of oxide were initially observed, but disappeared from the surface within a few hours. $\mathrm{Hg}$ oxide is expected to decompose due to the low oxygen partial pressure in this pseudo-UHV environment. ${ }^{14}$ In addition, we observe a continuous evaporation and recondensation of $\mathrm{Hg}$, constantly renewing the $\mathrm{Hg}$ surface. ${ }^{15}$ The resulting $\mathrm{Hg}$ surface was stable, as determined by x-ray reflectivity, for 11 days.

In both chambers, a copper cold finger extended from the bottom of the sample pan into a liquid nitrogen dewar. Temperature was monitored near the sample pan, and calibrated in separate experiments with a sensor immersed in the $\mathrm{Hg}$. Corrected sample temperatures are reported with relative errors of $\pm 1{ }^{\circ} \mathrm{C}$. For the UHV chamber, the gradient between sample and thermometer was greater, and there is an additional systematic error that may be as large as $8{ }^{\circ} \mathrm{C}$. Both chambers rested on an active vibration isolation unit.

Our x-ray reflectivity measurements reveal that the surface induced layering is strongly temperature dependent. Figure 1(a) shows the reflectivity as a function of $q_{z}$ measured in the glass chamber for three temperatures between $-35^{\circ} \mathrm{C}$ and room temperature. The quasi-Bragg peak observed at $q_{z} \approx 2.2 \AA^{-1}$ indicates that the liquid metal is stratified near the surface. The peak position, amplitude, and width give the length scales for the surface induced layering, which extends several $\AA$ into the bulk liquid. The other main feature of the data is the minimum in the reflectivity at $q_{z}$ $\approx 0.6-1.2 \AA^{-1}$, the depth of which differs between samples. This minimum is much more pronounced under the highpressure $\mathrm{H}_{2}$ environment (900 Torr) than under the $\mathrm{Hg}$ vapor pressure of $10^{-2}$ Torr, as shown for two sets of roomtemperature measurements in Fig. 1(b). These low- $q_{z}$ data indicate that the details of the electron density within $5 \AA$ of the surface are affected by the sample environment.

We have quantified the surface layering by constructing a model for the surface-normal density profile, parametrized as reported previously, ${ }^{3,4,8}$ and fitting the reflectivity calculated from the model to the experimental data. The surface-normal density profile is constructed from slabs of electron density parallel to the liquid-vapor interface. The slabs represent planes of atoms separated by a distance $d$, which are disordered in plane, and which have mean-squared surface-normal displacements increasing with depth. The electron density profile is composed of a sum of Gaussian terms:
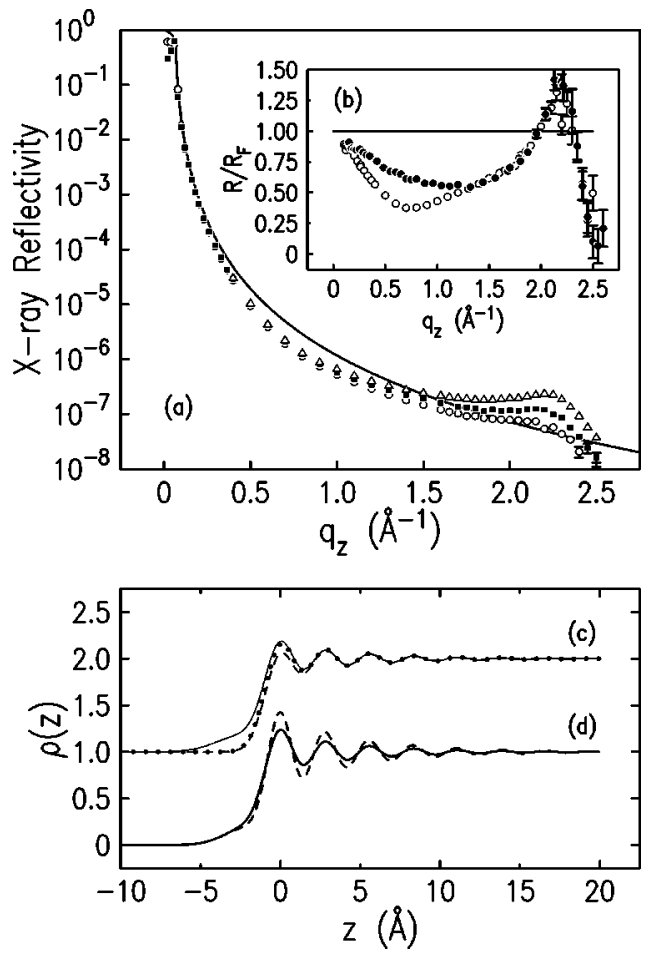

FIG. 1. (a) X-ray reflectivity of liquid $\mathrm{Hg} . \triangle:-35^{\circ} \mathrm{C}$; $\mathbf{\square}$ : $0{ }^{\circ} \mathrm{C}$; O: $+23{ }^{\circ} \mathrm{C}$; (-): calculated Fresnel reflectivity $R_{F}$. (b) Roomtemperature reflectivity normalized by $R_{F}(-)$ for two Hg samples. $\bigcirc$ : glass chamber with $\mathrm{H}_{2}$ gas. $\bigcirc$ : UHV chamber. (c) Calculated local layering profiles having $d=2.72 \AA, \bar{\sigma}=0.46 \AA$, and $\sigma_{T}=1.0$ $\AA$. $(\cdots)$ : no surface modification. (—): adlayer model. (- - -): surface depletion model. (d) Best-fit profiles for vacuum data. (-): Room temperature $\left(\sigma_{T}=1.0 \AA\right)$. $(---): T=-36{ }^{\circ} \mathrm{C}\left(\sigma_{T}=0.8 \AA\right)$.

$$
\rho(z)=\rho_{\infty} \sum_{n=0}^{\infty} \frac{d / \sigma_{n}}{\sqrt{2 \pi}} \exp \left[-(z-d)^{2} / 2 \sigma_{n}^{2}\right],
$$

where $\rho_{\infty}$ is the electron density of bulk $\mathrm{Hg}$, and we represent the mean-squared displacements in the $n$th layer by $\sigma_{n}^{2}$ $=n \bar{\sigma}^{2}+\sigma_{T}^{2}$, where $\sigma_{T}$ characterizes the roughness of the layer nearest the surface. Far into the bulk, $n \bar{\sigma}^{2}$ becomes large, and no layering is present, so that $\bar{\sigma}$ quantifies a layering decay length. With this form for $\rho(z)$, the reflectivity, proportional to the Fresnel reflectivity $R_{F}$ of an abruptly terminated homogeneous electron density, can be calculated analytically and yields

$$
\begin{aligned}
\frac{R\left(q_{z}\right)}{R_{F}}= & \left|\frac{1}{\rho_{\infty}} \int_{-\infty}^{+\infty}(\partial \rho / \partial z) \exp \left(i q_{z} z\right) d z\right|^{2} \\
= & F^{2}\left(q_{z} d\right)^{2} \exp \left(-\sigma_{T}^{2} q_{z}^{2}\right)\left[1-2 \exp \left(-q_{z}^{2} \bar{\sigma}^{2} / 2\right)\right. \\
& \left.\times \cos \left(q_{z} d\right)+\exp \left(-q_{z}^{2} \bar{\sigma}^{2}\right)\right]^{-1},
\end{aligned}
$$

where the reduced scattering form factor $F=\left(f\left(q_{z}\right)\right.$ $\left.+f^{\prime}\right) /\left(Z+f^{\prime}\right)$ has been inserted, as presented previously. ${ }^{16}$

The parameters $\sigma_{T}, \bar{\sigma}$, and $d$ control the height, width, and position of the layering peak, and characterize a simple local layering profile [Fig. 1(c), dotted line]. Although this model is sufficient for liquid Ga (Refs. 4 and 8) and In, ${ }^{5}$ it does not yield a good fit to the the low $-q_{z}$ reflectivity of liquid $\mathrm{Hg}$. To account for the low $-q_{z}$ reflectivity we have 
consideredprofiles that incorporate density terms in addition to the layered model, modifying the first $\mathrm{Hg}$ layer, the first several layers, and/or the region several $\AA$ towards the vapor side of the interface. These additional features are independent of temperature. The modification having the best fit for all data is a single additive Gaussian term $f_{A} \rho_{\infty}\left(d / \sigma_{A} \sqrt{2 \pi}\right) \exp \left[-\left(z-z_{A}\right) / 2 \sigma_{A}^{2}\right]$, positioned a few $\AA$ into the vapor region, with a width $\sigma_{A} \approx 1.5 \AA$ and a density $f_{A}$ relative to the bulk varying from $0.1-0.3$ between samples [Fig. 1(c), solid line]. However, this model is difficult to distinguish mathematically from one in which the density of the first $\mathrm{Hg}$ layer is decreased. In particular, the data with the most exaggerated low- $q_{z}$ minima can be fit by models with a broadened, depleted first $\mathrm{Hg}$ layer and an expanded spacing between the first two layers [Fig. 1(c), dashed line]. Models having alternating long and short layer spacings, as might result from atomic pairing, do yield destructive interference at low $q_{z}$ but do not fit the data well.

Deviations from the simple oscillatory profile are smaller for samples poured in a UHV chamber at $10^{-7}$ Torr following a bakeout than for measurements in a glass chamber evacuated to only $10^{-4}$ Torr. To explain this difference, we attribute the modified density profile to the presence of impurities. In recent self-consistent quantum Monte Carlo simulations of the $\mathrm{Hg}$-vapor interface, no vapor-side tail or appreciable modification of the surface layer was observed in the computed density profile. ${ }^{17}$ As discussed above, we believe the presence of macroscopic oxide is unlikely, although oxygen, water, or other contaminants introduced with the $\mathrm{Hg}$ sample may be present in small amounts. These may form a passivated, low-density adlayer or incorporate themselves into the first $\mathrm{Hg}$ layer to alter its density.

Although the available x-ray data do not allow a unique determination of the near-surface region, we obtain reliable information on the temperature dependence of the surfacenormal density profile. All $\mathrm{Hg}$ data are described by local layering parameters $d=2.72 \pm 0.02 \AA$ and $\bar{\sigma}=0.46 \pm 0.05 \AA$. These values are obtained for both $\mathrm{H}_{2}$ and vacuum measurements, and are independent of the model surface modification. The temperature dependence of the reflectivity can be quantified solely by the variation of $\sigma_{T}$, as shown in Fig. 2(a) for measurements in $\mathrm{H}_{2}$ atmosphere. This parameter controls the amplitude of the layering peak and is directly related to the amplitude of the density oscillations at the surface, as shown in Fig. 1(d) for vacuum measurements, varying from $0.8 \AA$ near the melting point to about $1.0 \AA$ at room temperature. The other parameters, if allowed to vary for each temperature independently, show no systematic dependence on $T$.

In particular, this means that the layering decay length of $5.0 \pm 0.5 \AA$ for $\mathrm{Hg}$ is independent of temperature, as was previously shown for $\mathrm{Ga}^{8}{ }^{8}$ This is somewhat surprising, since it is not obvious that layering should persist over essentially the same length scale in the face of thermal roughening that effectively broadens the interfacial width. Such a model, in which the local surface-normal density profile is independent of the surface height fluctuations, is intrinsic to the analyses long used for calculating the scattering cross section from rough surfaces. ${ }^{18,19}$ Previous observations on liquid metals demonstrated that such an interpretation appears to be justified. ${ }^{5,8}$ In fact, it has been thought that the liquid metal
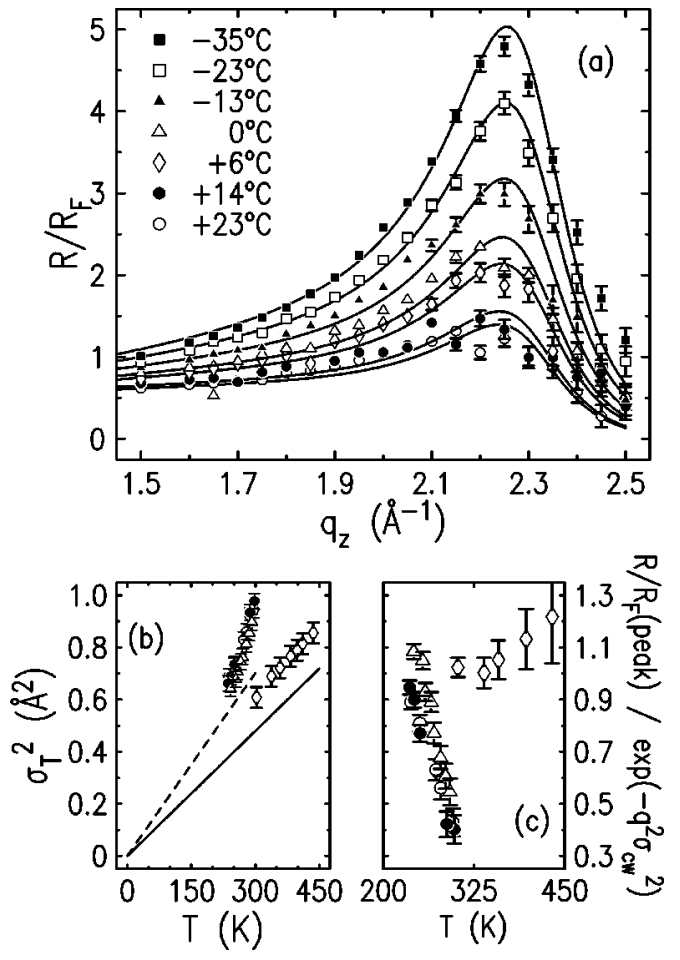

FIG. 2. (a) $R / R_{F}$ for different temperatures, from sample measured in $\mathrm{H}_{2}$ atmosphere. Solid lines are fit curves for which $\sigma_{T}$ has been varied. (b) Temperature dependence of $\sigma_{T}^{2} . \triangle$ : Measurement in vacuum. $\bigcirc$ : Measurement in $\mathrm{H}_{2}$ gas. $\bigcirc$ : Second sample measured in $\mathrm{H}_{2}$ gas. $\diamond: \mathrm{Ga}$ data from Ref. 8. Lines: Capillary wave form $\sigma_{c w}^{2}(T)$ for $\left.\mathrm{Hg}(--)^{-}\right)$and $\mathrm{Ga}(-)$. (c) Temperature dependence of $\left(R / R_{F}\right) / \exp \left(-q^{2} \sigma_{c w}^{2}\right)$ at the layering peak, normalized at the melting point, for $\mathrm{Ga}(\diamond)$ and $\mathrm{Hg}$ [symbols as in (b)].

surface may provide the best justification of this approach, since the measured reflectivity peak is a dramatic structural feature that is both straightforward to characterize and strongly affected by surface height fluctuations that vary considerably with temperature.

In the capillary wave model, thermally excited surface waves produce mean-squared displacements for which the restoring force is due to the surface tension $\gamma$ :

$$
\sigma_{\mathrm{cw}}^{2}(T)=\frac{k_{B} T}{2 \pi \gamma} \ln \left(\frac{k_{\mathrm{max}}}{k_{\mathrm{min}}}\right)
$$

where $k_{\max }=1.2 \AA^{-1}$ and $k_{\min }=7.4 \times 10^{-3} \AA^{-1}$ are wavevector cutoffs determined by the atomic size and the instrumental resolution, respectively. ${ }^{1}$ This model has been successfully applied to dielectric liquids ${ }^{1,20}$ and to liquid $\mathrm{Ga}^{8}$ Figure 2(b) compares $\sigma_{\mathrm{cw}}^{2}$ calculated as a function of temperature for $\mathrm{Hg}$ and $\mathrm{Ga}$ (dashed and solid lines), for appropriate values of the surface tension, ${ }^{21}$ bulk interatomic spacing, ${ }^{21}$ and instrumental resolution, to the surface roughness $\sigma_{T}^{2}$ extracted from reflectivity measurements of $\mathrm{Hg}$ and $\mathrm{Ga}$ (symbols). The temperature dependence of $\sigma_{T}$ differs fundamentally between $\mathrm{Hg}$ and $\mathrm{Ga}$. For $\mathrm{Ga}$, the slope of the experimental points matches that of the capillary wave model, offset only by a constant term $\sigma_{0} \approx 0.3 \AA$, attributed to an intrinsic roughness. ${ }^{8}$ This allows the quantity $\sigma_{T}$ for $\mathrm{Ga}$ (denoted in Ref. 8 by $\sigma_{c}$ ) to be written as $\sigma_{T}^{2}=\sigma_{0}^{2}+\sigma_{\mathrm{cw}}^{2}$. 
Liquid $\mathrm{Ga}$, therefore, has a temperature-independent local surface layering profile, roughened by capillary waves.

By contrast, $\sigma_{T}^{2}(T)$ for $\mathrm{Hg}$ has a much steeper slope than predicted by capillary wave theory, exhibiting an additional temperature-dependent roughness. This result is independent of the local layering model, as shown in Fig. 2(c), where we plot $\left(R / R_{F}\right) / \exp \left(-q^{2} \sigma_{\mathrm{cw}}^{2}\right)$ for $\mathrm{Hg}$ and $\mathrm{Ga}$ at their layering peaks vs $T$, normalized at the melting point. The Ga data increase only slightly with $T$, within their scatter of about $15 \%$. The $\mathrm{Hg}$ data exhibit a steep slope far exceeding the $\approx 15 \%$ scatter. The temperature dependence of the $\mathrm{Hg}$-vapor interface roughness has also been extracted from recent selfconsistent quantum Monte Carlo simulations. ${ }^{17}$ While similar linear behavior of $\sigma_{T}^{2}(T)$ is observed, direct comparison of the slope is complicated by the differences in capillary wave cutoffs and surface tension between the experimental and simulation samples.

The temperature dependence of $\gamma$, even considering the possible effects of impurities, can be ruled out as a cause. For pure $\mathrm{Hg}, d \gamma / d T \approx-0.2 \times 10^{-3} \mathrm{~N} / \mathrm{mK},{ }^{21}$ an order of magnitude too small to explain our observations. Optical measurements on $\mathrm{Hg}$ surfaces with varying degrees of cleanliness have found $d \gamma / d T$ in the range of $-0.7 \times 10^{-3}$ to
$+0.3 \times 10^{-3} \mathrm{~N} / \mathrm{mK}$ depending on the method of sample preparation. $^{22}$ The observation that oxide or contamination can produce $d \gamma / d T>0$ for $\mathrm{Hg}$ is notable, but this would cause a reduction of $\sigma_{T}$ with increasing $T$ rather than the increase observed, and so cannot account for our results.

We conclude that the surface structure of liquid $\mathrm{Hg}$ has a temperature-dependent component in addition to capillary wave roughening. This may take the form of temperaturedependent height fluctuations that increase the mean-squared atomic displacements beyond the effect of capillary waves, or of a temperature-dependent local density profile. The specular reflectivity measurements presented here cannot distinguish between these two cases. Measurements of diffuse scattering from the $\mathrm{Hg}$ surface may provide further information. These results reemphasize the fact that roughness intrinsic to the surface-normal density profile in general cannot be unambiguously separated from height fluctuations, despite previous treatments of scattering from the liquid surface.

This work was supported by U.S. DOE Grant Nos. DEFG02-88-ER45379 and DE-AC02-98CH10886, NSF Grant No. DMR-94-00396, and the U.S.-Israel Binational Science Foundation, Jerusalem. H.T. acknowledges support from the Deutsche Forschungsgemeinschaft.
*Author to whom correspondence should be addressed. Electronic address: dimasi@bnl.gov

${ }^{1}$ A. Braslau, P. S. Pershan, G. Swislow, B. M. Ocko, and J. AlsNielsen, Phys. Rev. A 38, 2457 (1988).

${ }^{2}$ S. A. Rice, J. Non-Cryst. Solids 205-207, 755 (1996).

${ }^{3}$ O. M. Magnussen, B. M. Ocko, M. J. Regan, K. Penanen, P. S. Pershan, and M. Deutsch, Phys. Rev. Lett. 74, 4444 (1995).

${ }^{4}$ M. J. Regan, E. H. Kawamoto, S. Lee, P. S. Pershan, N. Maskii, M. Deutsch, O. M. Magnussen, B. M. Ocko, and L. E. Berman, Phys. Rev. Lett. 75, 2498 (1995).

${ }^{5}$ H. Tostmann, E. DiMasi, P. S. Pershan, B. M. Ocko, O. G. Shpyrko, and M. Deutsch (to be published).

${ }^{6}$ H. Tostmann, E. DiMasi, O. G. Shpyrko, P. S. Pershan, B. M. Ocko, and M. Deutsch, Ber. Bunsenges. Phys. Chem. 102, 1136 (1998).

${ }^{7}$ N. Lei, Z. Huang and S. A. Rice, J. Chem. Phys. 104, 4802 (1996); 107, 4051 (1997); M. J. Regan, P. S. Pershan, O. M. Magnussen, B. M. Ocko, M. Deutsch, and L. E. Berman, Phys. Rev. B 55, 15874 (1997).

${ }^{8}$ M. J. Regan, P. S. Pershan, O. M. Magnussen, B. M. Ocko, M. Deutsch, and L. E. Berman, Phys. Rev. B 54, 9730 (1996).

${ }^{9}$ M. J. Regan, O. M. Magnussen, E. H. Kawamoto, P. S. Pershan, B. M. Ocko, N. Maskil, M. Deutsch, S. Lee, K. Penanen, and L. E. Berman, J. Non-Cryst. Solids 205-207, 762 (1996).

${ }^{10} \mathrm{D}$. Turnbull (private communication). More recently it has been recognized that passivation of $\mathrm{Hg}$ oxide occurs only at elevated temperatures: V. Kolevzon (private communication).

${ }^{11}$ The reflectivity measured for $q_{z} \gtrsim 1.2 \AA^{-1}$ varies by a few per- cent depending on the choice of vertical detector resolution, due to diffuse scattering near the specular condition that arises from surface fluctuations. We have not incorporated this small effect into our analysis of the reflectivity. A detailed discussion is given in Ref. 5.

${ }^{12}$ Quadruple distilled $\mathrm{Hg}$ with $<1 \mathrm{ppm}$ metal impurities, Bethlehem Apparatus Co., Inc., Front and Depot Streets, Hellertown, PA 18055.

${ }^{13}$ H. Tostmann, E. DiMasi, B. M. Ocko, M. Deutsch, and P. S. Pershan, J. Non-Cryst. Solids (to be published).

${ }^{14}$ B. N. Thomas, S. W. Barton, F. Novak, and S. A. Rice, J. Chem. Phys. 86, 1036 (1987).

${ }^{15}$ B. C. Lu and S. A. Rice, J. Chem. Phys. 68, 5558 (1978).

${ }^{16}$ In this paper, we consistently use $n=0$ to refer to the surface layer. This produces discrepancies with the equations given in Ref. 3.

${ }^{17}$ D. S. Chekmarev, M. Zhao, and S. A. Rice, Phys. Rev. E (to be published).

${ }^{18}$ S. K. Sinha, E. B. Sirota, S. Garoff, and H. B. Stanley, Phys. Rev. B 38, 2297 (1988).

${ }^{19}$ M. K. Sanyal, S. K. Sinha, K. G. Huang, and B. M. Ocko, Phys. Rev. Lett. 66, 628 (1991).

${ }^{20}$ M. Fukuto, R. K. Heilmann, P. S. Pershan, J. A. Griffiths, S. M. Yu, and D. A. Tirrell (unpublished).

${ }^{21}$ T. Iida and R. I. L. Guthrie, The Physical Properties of Liquid Metals (Oxford University Press, Oxford, 1993).

${ }^{22}$ V. Kolevzon and G. Pozdniakov, J. Phys.: Condens. Matter 9, 6815 (1997), and references therein. 